\title{
Exploiting the Potential of Digital Fabrication for Sustainable and Economic Concrete Structures
}

\section{Conference Paper}

\section{Author(s):}

Mata Falcón, Jaime (D); Bischof, Patrick; Kaufmann, Walter (D)

Publication date:

2018-09

Permanent link:

https://doi.org/10.3929/ethz-b-000297642

Rights / license:

In Copyright - Non-Commercial Use Permitted

Originally published in:

RILEM Bookseries 19, https://doi.org/10.1007/978-3-319-99519-9_14 


\title{
Exploiting the potential of digital fabrication for sustainable and economic concrete structures
}

\author{
J. Mata-Falcón* ${ }^{1[0000-0001-8701-4410]}$, P. Bischof ${ }^{1}$ and W. Kaufmann ${ }^{1}$ \\ ${ }^{1}$ Institute of Structural Engineering, Department of Civil, Environmental and Geomatic Engi- \\ neering, ETH Zurich \\ *Corresponding author; E-mail: mata-falcon@ibk.baug.ethz.ch
}

\begin{abstract}
Digital technologies overcome typical constraints of traditional concrete construction processes caused by the high impact of labour costs and bring about many new possibilities to the conceptual design, dimensioning, detailing, and production of concrete structures. While the potential of geometric flexibility is being extensively explored, most digital technologies encounter difficulties in penetrating the market due to lacking compliance with structural integrity requirements. To maximise their impact, it is essential that digital concrete processes (i) integrate reinforcement resisting tensile forces and (ii) address conventional structures with geometric simplicity. This paper discusses the potential of digital concrete fabrication processes to reduce the quantity of reinforcement required in concrete structures. For example, "minimum reinforcement" can be tremendously reduced by (i) tailoring the concrete grade locally to the actual needs and (ii) ensuring small crack spacings and correspondingly reduced crack widths by means of crack initiators. An experimental study shows that the strength reduction in the interfaces between layers from extrusion processes can be quantified with reasonable accuracy, which allows using these weak interfaces as crack initiators. A mechanical model to quantify the corresponding potential for saving "minimum reinforcement" when using $3 \mathrm{D}$ printing is presented. It is found that weak interfaces in layer joints with $33 \%$ of the concrete tensile strength inside the layer allow reducing up to $80 \%$ the minimum reinforcement for a given maximum crack width requirement under imposed deformations.
\end{abstract}

Keywords: Digital fabrication, concrete structures, minimum reinforcement, sustainability, durability, 3D printing.

\section{Introduction}

Reinforced concrete is by far the most used building material today. Existing concrete and reinforcement technology and approaches for its dimensioning have been optimised for more than a century hand in hand with traditional construction methods. This has led to highly efficient conventional concrete construction processes, hard to beat by novel technologies. 


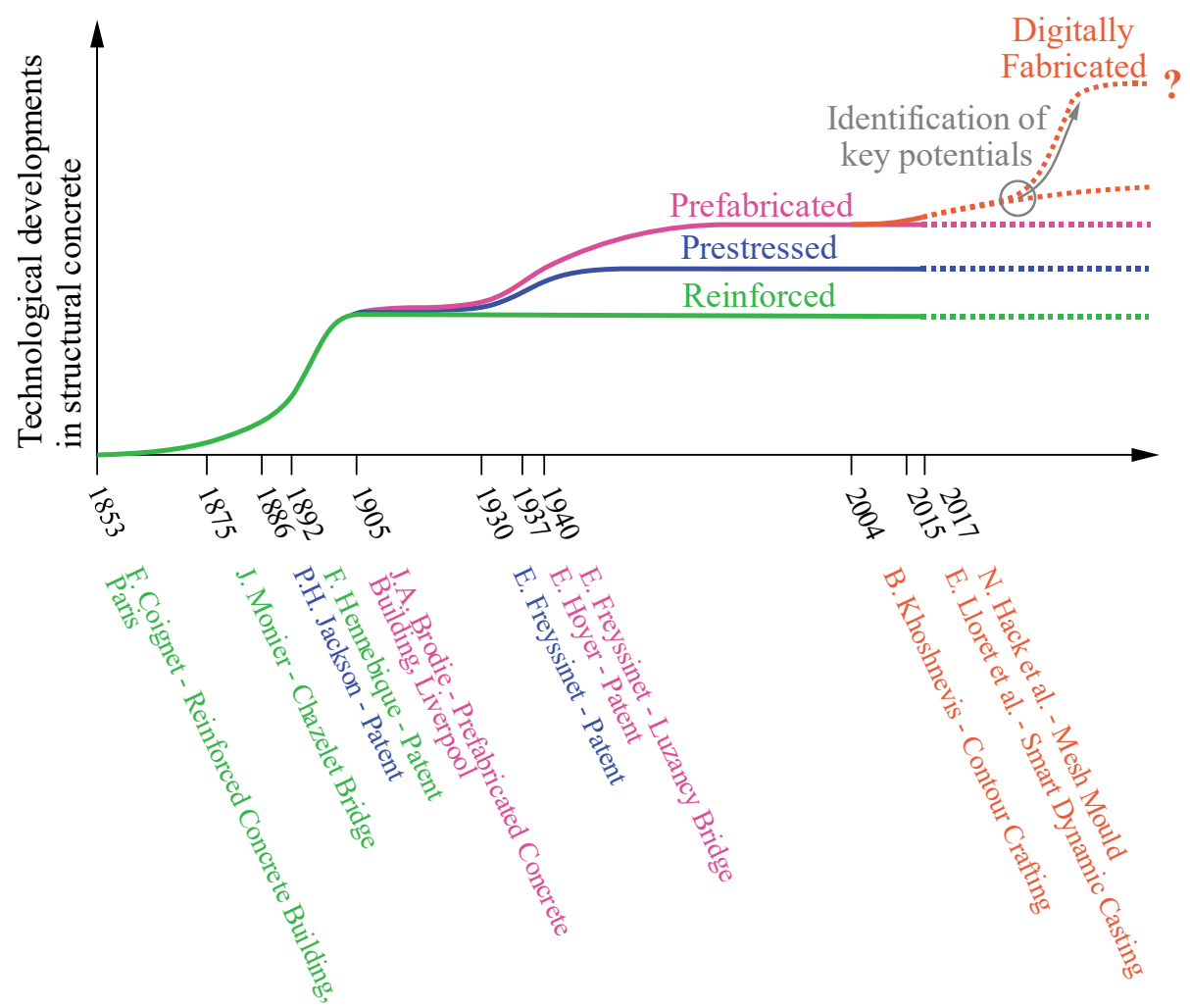

Fig. 1. Technological developments in structural concrete

The implementation of digital technologies brings about many new possibilities for the conceptual design, dimensioning, detailing, and production of concrete structures, promising to revolutionise the concrete construction industry. Over the past years, the digital fabrication research community has developed diverse interesting digital fabrication technologies with strong focus on materials and geometrical aspects [1]-[5]. These technologies have the potential of overcoming typical constraints of traditional processes caused by the high impact of labour costs. Nevertheless, even the most promising processes are currently only competitive for special applications and encounter difficulties in penetrating the market due to high costs and lacking compliance with structural integrity requirements of clients and building codes. Therefore, digital fabrication for large-scale and mass-market concrete structures is a persisting challenge.

The difficulties of digital fabrication inventions to penetrate the market are analogous to the obstacles found by historical key developments in structural concrete. The graph in Fig. 1 shows that after invention of new concepts, several decades of research and small scale applications passed before the concepts reached maturity with ideas how to exploit their potential. Only then, the key developments of reinforced concrete, prestressed concrete and prefabrication were adopted by mass market.

In general, the conceptual design, dimensioning, detailing, and production of concrete structures should be systematically re-thought to assess the potential of digital 
fabrication. Benefits that digital fabrication brings to structural concrete need to be identified at early development stage in order to exploit the full potential offered by these new technologies to build economic, code-compliant and sustainable concrete structures. The present contribution discusses some intrinsic benefits of digital concrete fabrication related to reducing the required quantity of reinforcement.

\section{Requirements of mass-market concrete structures}

\section{$2.1 \quad$ Necessity of reinforcement}

Current 3D printing by layered extrusion (e.g. "contour crafting") - the most popular digital fabrication technology with concrete today- typically does not allow adding reinforcement during the production. The use of reinforcement resisting tensile forces is, however, essential for the load bearing capacity of structural concrete, since concrete tensile strength is unreliable and therefore typically neglected in structural design. Moreover, reinforcement is not only required to provide strength: A "minimum reinforcement" is always required to (i) avoid brittle failures at cracking, (ii) ensure a sufficiently ductile behaviour to enable plastic redistribution, and (iii) limit deformations and crack widths in real-life structures. The first two functions ensure a reasonable deformation capacity, which is required to apply standard bearing capacity verifications according to design codes for structural concrete. The third function of minimum reinforcement addresses the behaviour under service conditions and durability, which governs the overall reinforcement quantity in many structures. In conclusion, 3D printed elements without reinforcement are essentially suitable only as a replacement of unreinforced masonry, which could be erected much more efficiently both conventionally as well as using robots [6].

While the combined use of external post-tensioned reinforcement and fibre-reinforced concrete can significantly improve the structural performance of 3D printed concrete elements, their strength and ductility will still be limited with respect to conventionally reinforced concrete structures. Hence, the integration of internal reinforcement in $3 \mathrm{D}$ printing is fundamental to produce load bearing concrete structures more efficiently than with traditional construction methods. In some digital fabrication techniques, such as Smart Dynamic Casting [3] and Mesh Mould [4] processes, this requirement has already been implemented.

\subsection{Limited use of geometrical complexity}

The current building stock almost completely consists of rectangular buildings, as summarised by Steadman [7] from two exemplary studies. With digital fabrication technologies, complex geometries may be produced in some cases with a similar production effort than simpler geometries. Hence, digital concrete fabrication could overcome the restriction caused by rectangular building components in existing technologies. This flexibility brings form optimization design processes into a new dimension, allowing using structurally optimum shapes and minimizing the use of materials for a certain 
structural element at the same production cost. While this is a powerful benefit, its potential will most likely not be fully exploited in mass market concrete structures, because of other requirements of geometric simplicity independent of the building technology, as pointed out e.g. by Steadman [7] who elaborated reasons for vertical and horizontal rectangularity of buildings: Verticality is structurally efficient as typically dominant gravity loads are transferred directly to the ground, and horizontal flat slabs and straight walls serve to exploit the building space. Furthermore, flat floors permit easy manoeuvring and placing of, usually rectangular, furniture, and horizontal rectangularity provides unlimited possibilities to divide buildings and rooms and freedom to pack next to each other. While some of these requirements may not apply to all buildings (e.g. in single spaces such as theatres or museums non-rectangularity is frequent since close packing is not required), geometric simplicity will be still required in most cases. Hence, it is important to find efficient digital construction processes for these standard applications.

\section{Benefits of digital fabrication for structural concrete}

\subsection{Key potentials}

Besides the geometric flexibility, whose use may be limited as outlined above, digital fabrication brings about many potentials that should be exploited while aiming at conforming to mass-market requirements.

The robotic placement of reinforcement permits providing reinforcement in any direction and length in the optimum directions and strictly in the statically required amount, significantly reducing the reinforcement quantities for ultimate limit state, especially in walls and slabs. Furthermore, it has the potential of reducing on-site adjustments to the planned reinforcement due to limitations on manual placement and hazards and health risks of construction workers (e.g. lifting heavy rebar and fall hazards of steel fixers).

Equally promising is a reduction of minimum reinforcement, which often makes up for more than $50 \%$ of the total reinforcement content. The required amount of minimum reinforcement to avoid brittle failures at cracking (see subsection 2.1) increases proportionally to the cracking load of the structure, which is directly dependent on the concrete tensile strength. Hence, by tailoring the concrete grade locally to the actual needs by digital fabrication processes, i.e. reducing the cement content where a low concrete strength is sufficient, the corresponding minimum reinforcement could be reduced very significantly (in addition to savings in cement content). Another process that could tremendously reduce the minimum reinforcement is the fabrication of crack initiators by digital technologies. These crack initiators would (i) reduce the cracking load and (ii) ensure small crack spacings and correspondingly reduced crack widths. Both aspects contribute to reducing the minimum reinforcement. Crack initiators could be easily produced by means of geometric or material discontinuities introduced in digital fabrication technologies. One example of intrinsic crack initiators caused by material 
discontinuities are the weak interfaces between concrete layers in 3D printing processes. These weak interfaces, intuitively perceived as a disadvantage of 3D concrete printing, can be used either to reduce the reinforcement content or to improve the durability of the structures as will be further discussed in the subsection 3.2.

\subsection{Crack initiators by 3D printing weak interfaces}

\section{Relevance}

Weak interfaces between layers cast at different time have been widely reported in the literature. For example, Roussel and Cussigh [8] reported on the formation of distinct layers (also designated as "cold joint"), which may occur in interfaces of Self Compacting Concrete (SCC) depending on the delay between the casting of layers. The occurrence of distinct layers "depends strongly on the thixotropic behaviour of the SCC, the thickness of the layers and on the roughness of the interface between the two layers" [8]. Zareiyan and Khoshnevis [9] experimentally analysed the bond strength between layers cast after each other by means of compression and splitting tests. This study showed that a casting interval of 12 minutes with layers of $50.8 \mathrm{~mm}$ thickness caused a reduction of the tensile strength (measured at an age 28 days) of about $10 \%$.

Due to shrinkage and temperature strains, concrete is prone to cracking regardless of the amount of reinforcement in the structure and the presence of mechanical actions. In engineering practice, reduced crack widths are relevant for surfaces directly exposed to the environment for reasons of water tightness, durability and/or aesthetics (fair-faced concrete). Designers typically increase the reinforcement content to reduce the crack spacing and hence the crack widths. By using weak interfaces as crack initiators, the crack spacing and crack widths could be reduced without increased reinforcement content.

The following sections present (i) an experimental study to assess the reduction of the bending tensile strength that can be achieved in layered casting processes, as well as (ii) a mechanical model to quantify the potential saving of minimum reinforcement when using $3 \mathrm{D}$ printing weak interfaces.

\section{Pilot experiments}

In a pilot experimental study by the authors, three-point bending flexural tests on 60 specimens of $80 \mathrm{~mm} \times 80 \mathrm{~mm} \times 270 \mathrm{~mm}$ with a span of $200 \mathrm{~mm}$ were conducted. The specimens were cast vertically in two steps (approx. $135 \mathrm{~mm}+135 \mathrm{~mm}$ ) at room temperature. The casting interval between layers was varied $(\min .3$ specimens per casting interval). The study aimed at examining whether it is possible to regulate the concrete tensile strength $f_{c t}$ of a specific type of concrete in the interface between layers.

The concrete was similar to the one used for Smart Dynamic Casting projects [3], which is similar to the standard formulations used in 3D printing processes. The mix was slightly modified in order to increase the flowability and, hence, to easily level the layer surface. A level layer surface improves the repeatability of the interface tensile strength. 


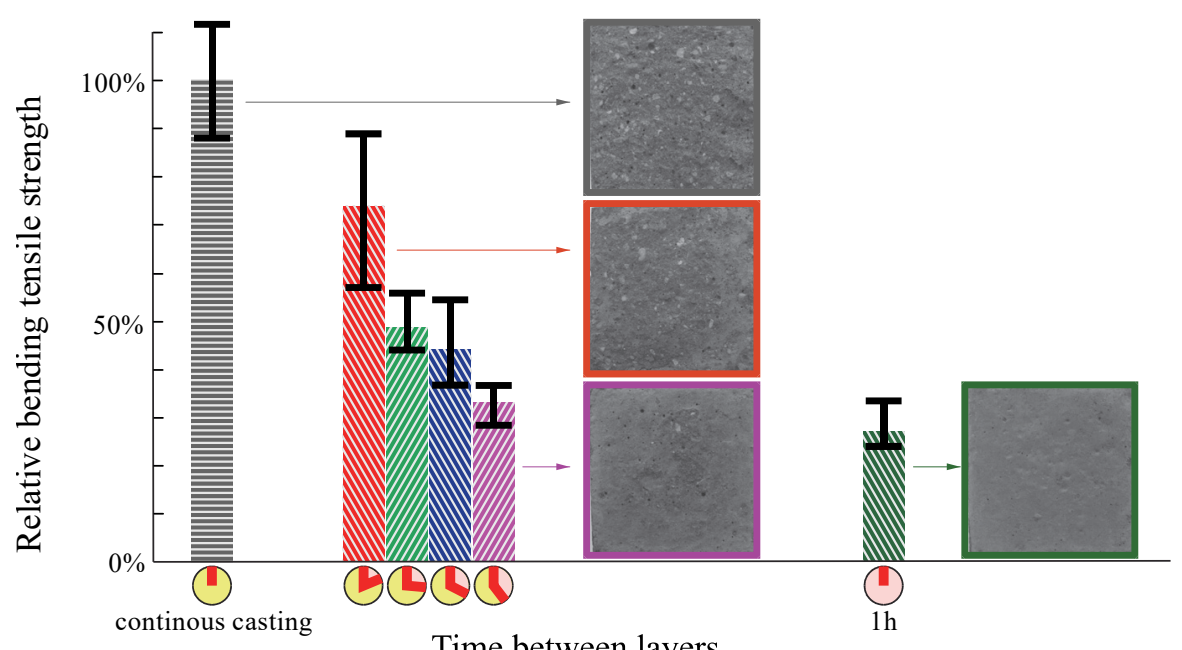

Time between layers

Fig. 2. Bending tensile strength of weak interfaces and its scatter for varying casting intervals normalised with respect to the reference specimens with continuous casting, and photos of failed interfaces.

Fig. 2 shows the bending tensile strength in average and in its scatter (minimum to maximum) of weak interfaces with varying casting intervals in relationship to the reference specimens (continuous casting) after 21 days. Furthermore, photos of the failed weak interfaces are shown, of the reference specimen and of the specimens with 12minutes, 24-minutes and 1-hour casting interval between layers. The results show that the loss of bending tensile strength in a weak interface greatly evolves between $10 \mathrm{~min}$ and $30 \mathrm{~min}$ for the used type of concrete. For intervals greater than $2 \mathrm{~h}$, the two parts of the specimens had no adherence at all.

\section{Reduction of minimum reinforcement}

Crack widths in concrete structures are typically calculated by multiplying the crack spacing with the average tensile strain of the reinforcement $\left(w_{r}=s_{r} \varepsilon_{s m}\right)$, neglecting the typically small reduction of the crack widths caused by the tensile strains of the concrete between the cracks. Crack spacings as well as tensile strains and stresses between the cracks can be determined from basic mechanical principles using e.g. the Tension Chord Model (TCM) [10]. In this section, the TCM is modified to consider the effect of weak interfaces between 3D printing layers in order to quantify the reduction of the crack width and/or the reduction of the reinforcement allowed by the presence of crack initiators (subscript wi used for "weak interfaces" in the following).

The main assumption of the TCM is the consideration of a stepped, rigid-perfectly plastic bond shear stress-slip relationship with bond strengths $\tau_{b}=\tau_{b 0}=2 f_{c t}$ prior to yielding, and $\tau_{b}=\tau_{b 1}=f_{c t}$ after onset of yielding. Hence, at serviceability load levels where the reinforcement remains elastic, constant bond stresses are considered, similar to the simplification adopted by many design codes. While cracks are considered as stress free, tensile stresses are transferred from reinforcement to concrete between 
(a)

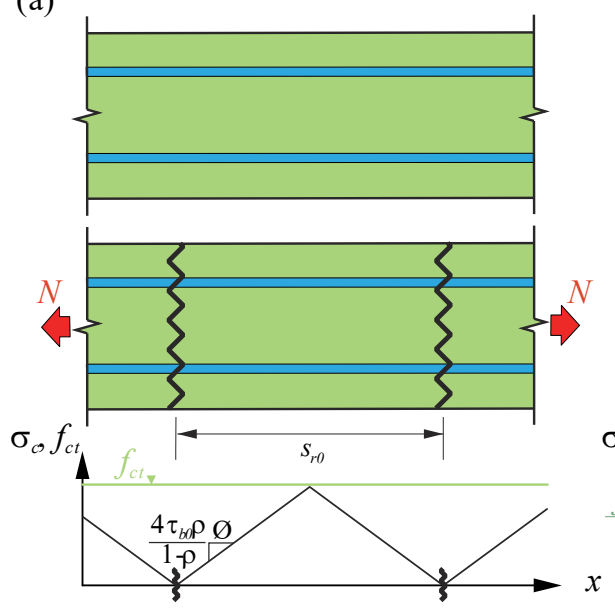

(b)
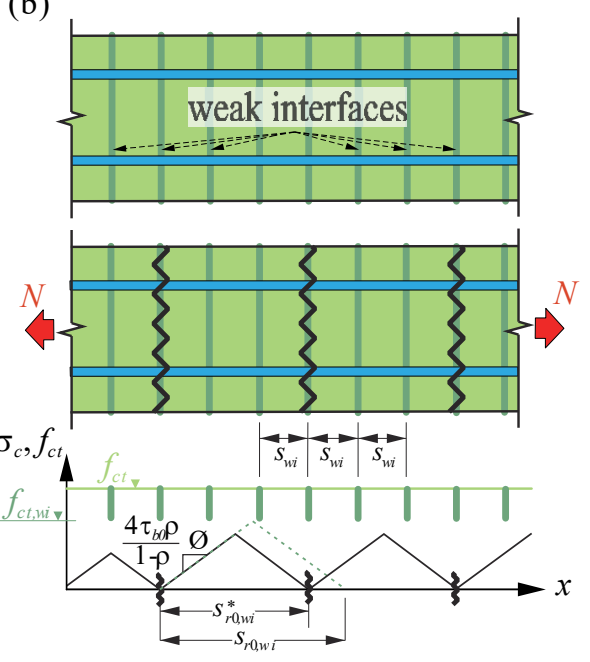

Fig. 3. Tension chords with maximum crack spacing and distribution of concrete tensile stresses: (a) TCM for a continuously cast element; (b) modified TCM considering weak interfaces.

cracks by bond shear stresses. The maximum spacing between two cracks $\left(s_{r 0}\right)$ will be produced when the tensile strength of concrete $\left(f_{c t}\right)$ is reached at the centre between two cracks (Fig. 3a). For the modified TCM presented here, considering crack initiators by 3D printing weak interfaces (Fig. 3b), the layering process is assumed to affect exclusively the concrete tensile strength in the weak interfaces $\left(f_{c t, w i}\right)$, which is reduced as follows:

$$
f_{c t, w i}=\xi \cdot f_{c t}
$$

The authors are currently conducting an experimental study to evaluate whether the layering process could also influence the bond strength $\tau_{b 0}$ and $\tau_{b 1}$.

Under these assumptions, and for layer thicknesses $\left(s_{w i}\right)$ considerably smaller than the crack spacing without weak layers (as usually happens for standard 3D printing processes, e.g. $\left.s_{w i}=20 \mathrm{~mm}, s_{w i} / s_{r 0} \approx 0.1\right)$ the maximum crack spacing $s_{r 0, w i}$ of a tension chord incorporating weak interfaces is determined by:

$$
s_{r 0, w i}=\frac{f_{c t, w i} \varnothing(1-\rho)}{2 \tau_{b 0} \rho}=\xi \cdot s_{r 0}
$$

where $\varnothing$ is the diameter of the reinforcement, $\rho$ is the geometric reinforcement ratio of the tension chord and $s_{r 0}$ is the maximum crack spacing in an element without crack initiators.

The presence of weak interfaces reduces the maximum crack spacing proportionally to the reduction of the concrete tensile strength in the interfaces between layers $(\xi)$. The cracking load and the steel stresses at the crack at the onset of cracking $\left(\sigma_{s, c r}\right)$ are also reduced proportionally to the relative strength in the interfaces: 


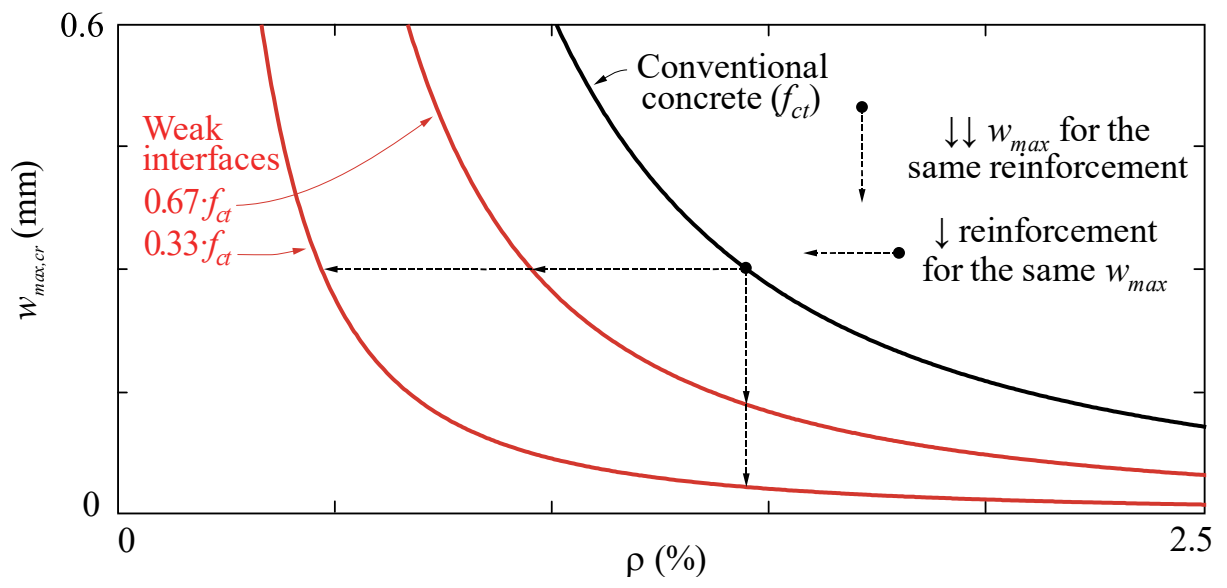

Fig. 4. Impact of weak joints between layers of extrusion processes on the amount of reinforcement required to control the maximum crack width caused by imposed deformations, assuming thin layers $\left(\varnothing=18 \mathrm{~mm} ; f_{c t}=4.8 \mathrm{MPa}\right)$.

$$
\sigma_{s r, c r, w i}=\xi \cdot f_{c t}\left(\frac{1}{\rho}-1+\frac{E_{s}}{E_{c}}\right)=\xi \cdot \sigma_{s r, c r}
$$

where $E_{s}$ and $E_{c}$ are the Young's moduli of steel and concrete respectively.

The maximum crack width at the onset of cracking $\left(w_{\max , c r}\right)$, relevant for durability verifications in elements subjected to imposed deformations, can be estimated as follows in the presence of weak interface crack initiators:

$$
w_{\text {max }, c r, w i}=s_{r 0, w i}\left(\frac{\sigma_{s r, c r, w i}}{E_{s}}-\frac{\tau_{b 0} s_{r 0, w i}}{\varnothing E_{s}}\right)=\xi s_{r 0}\left(\frac{\xi \sigma_{s r, c r}}{E_{s}}-\frac{\tau_{b 0} \xi_{r 0}}{\varnothing E_{s}}\right)=\xi^{2} \cdot w_{\text {max }, c r}
$$

The effect of weak interfaces in layering processes allows reducing either the maximum crack width or the reinforcement content required for a certain maximum crack width requirement. As Fig. 4 shows for an example tension chord with reinforcement of $\varnothing 18 \mathrm{~mm}$, weak interfaces with a $33 \%$ of the concrete tensile strength inside the layer allow reducing by $67 \%$ the minimum reinforcement required to avoid brittle failures at cracking (reduction quasi-proportional to the reduction of the tensile strength in the weak interfaces, $\xi$ ). At the same time, these weak layer joints would allow reducing by around $90 \%$ the maximum crack with a constant reinforcement content (reduction proportional to the square of the reduction of the tensile strength in the weak interfaces, $\left.\xi^{2}\right)$. Hence, the possibility to introduce controlled crack initiators has a huge potential to reduce very significantly the amount of minimum reinforcement and/or improve the durability of many reinforced concrete structures.

In real-life structures, a reduction of the amount of minimum reinforcement has additional benefits for the crack control since it allows reducing the diameter of the reinforcement when the reinforcement spacing is fixed, e.g. because of constructive reasons. In such cases, using the assumptions of the TCM, the crack width is directly proportional to the diameter of the reinforcement. Hence, the amount of minimum reinforcement can be further reduced for a certain crack width requirement. This effect is 


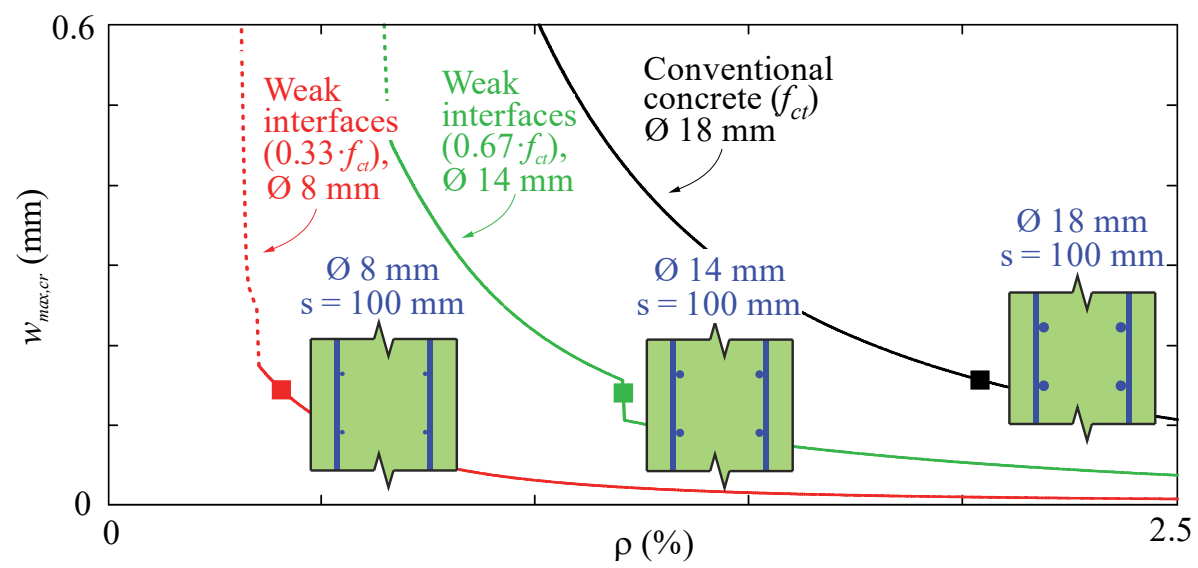

Fig. 5. Example of the required longitudinal reinforcement content for a maximum crack width requirement of $0.15 \mathrm{~mm}$ considering a conventional concrete wall and concrete walls with weak interfaces between layers $\left(f_{c t}=4.8 \mathrm{MPa} ; s_{w i}=100 \mathrm{~mm}\right)$. The dashed line indicates yielding of reinforcement at cracking.

shown in Fig. 5 with a fixed longitudinal reinforcement spacing of $100 \mathrm{~mm}$. In this case, weak joints with $33 \%$ of the concrete tensile strength inside the layer allow reducing by $80 \%$ the minimum reinforcement. Since this example considers a layer thickness $\left(s_{w i}\right)$ in the same order of magnitude as the crack spacing, the simplification considered in Eq. (2) cannot be directly adopted and the maximum crack spacing has to be rounded to an odd number of layers $\left(s_{r o, w i}^{*}\right)$, as shown in Fig. $3 \mathrm{~b}$.

\section{Conclusion and outlook}

Digital technologies are extensively exploring the potential of geometric flexibility. Nevertheless, most of them encounter difficulties in penetrating the market due to lacking compliance with structural integrity requirements. To maximise their impact, is essential that digital concrete processes (i) integrate reinforcement resisting tensile forces and (ii) address conventional structures without geometrical complexity.

Digital concrete fabrication processes have a great potential to reduce the quantity of reinforcement required in concrete structures even for standard geometries. The robotic placement of reinforcement permits providing reinforcement in the statically required amount. Moreover, minimum reinforcement can be tremendously reduced by (i) tailoring the concrete grade locally to the actual needs and (ii) ensuring small crack spacings and correspondingly reduced crack widths by means of crack initiators.

The results of an experimental pilot study show that the strength reduction in the interfaces between layers from extrusion processes can be quantified depending on the casting interval of the layers. This allows using these weak interfaces as crack initiators. A mechanical model to quantify the potential for saving minimum reinforcement when using these crack initiators generated in $3 \mathrm{D}$ printing is presented. It is found that weak 
interfaces in layer joints with $33 \%$ of the concrete tensile strength inside the layer allow reducing up to $80 \%$ the minimum reinforcement for a given maximum crack width requirement against imposed deformations.

Current research of the authors is addressing beneficial effects of weak interfaces and other intrinsic characteristics of digitally fabricated concrete structures aiming at exploiting them for developing sustainable and economic products and production processes able to penetrate the mass market.

\section{Acknowledgements}

This research is supported by the National Centre for Competence in Research in Digital Fabrication in Architecture, funded by the Swiss National Science Foundation (project number 51NF40_141853).

The authors would like to thank Lex Reiter (IFB, ETH Zürich) for his indispensable support for the planning and the conduction of the presented experiments.

\section{References}

[1] B. Khoshnevis, "Automated construction by contour crafting - related robotics and information technologies," Autom. Constr., vol. 13, no. 1, pp. 5-19, Jan. 2004.

[2] R. A. Buswell, R. C. Soar, A. G. F. Gibb, and T. Thorpe, "Freeform construction: megascale rapid manufacturing for construction," 2007.

[3] E. Lloret et al., "Complex concrete structures: Merging existing casting techniques with digital fabrication," Comput.-Aided Des., vol. 60, pp. 40-49, Mar. 2015.

[4] N. Hack et al., "Mesh Mould: An On Site, Robotically Fabricated, Functional Formwork," presented at the Second Concrete Innovation Conference (2nd CIC), Tromsø, Norway, 2017.

[5] T. Wangler et al., "Digital Concrete: Opportunities and Challenges," RILEM Tech. Lett., vol. 1, pp. 67-75, Oct. 2016.

[6] V. Helm, S. Ercan, F. Gramazio, and M. Kohler, "In-situ robotic construction: Extending the digital fabrication chain in architecture," in Proceedings of the 32nd Annual Conference of the Association for Computer Aided Design in Architecture (ACADIA), San Francisco, 2012, pp. 169-176.

[7] P. Steadman, "Why are most buildings rectangular?," Archit. Res. Q., vol. 10, no. 2, pp. 119-130, 2006.

[8] N. Roussel and F. Cussigh, "Distinct-layer casting of SCC: The mechanical consequences of thixotropy," Cem. Concr. Res., vol. 38, no. 5, pp. 624-632, May 2008.

[9] B. Zareiyan and B. Khoshnevis, "Interlayer adhesion and strength of structures in Contour Crafting - Effects of aggregate size, extrusion rate, and layer thickness," Autom. Constr., vol. 81, pp. 112-121, Sep. 2017.

[10] P. Marti, M. Alvarez, W. Kaufmann, and V. Sigrist, "Tension Chord Model for Structural Concrete," Struct. Eng. Int., vol. 8, pp. 287-298, 1998. 